\title{
Incorporação de fonte de nitrogênio em partículas de PVA e alginato de sódio e estudo da influência de ciclos de congelamento/descongelamento na caracterização do produto
}

\section{A source of nitrogen incorporation in particulate PVA and sodium alginate and study of the influence of freezing/thawing cycles in the characterization of the product}

\author{
Sinara Queli Welter Nardi ${ }^{*}$, Sirlei Dias Teixeiraํㄹ e Cristiane Regina Budziak Parabocz ${ }^{1}$ \\ 'Departamento de Química, Universidade Tecnológica Federal do Paraná - UTFPR, \\ Pato Branco, PR, Brasil \\ *welter.sinara@gmail.com
}

\section{Resumo}

Neste trabalho foram incorporadas duas fontes de nitrogênio (ureia e caulinita intercalada com ureia) em matriz polimérica de álcool polivinílico e alginato de sódio na proporção de 3:1, utilizando a metodologia de gotejamento em solução de $\mathrm{CaCl}_{2}$. As partículas foram submetidas ao congelamento e posterior descongelamento com o intuito de melhorar a estrutura e resistência térmica da matriz polimérica. As partículas foram caracterizadas através de Análise Elementar, FTIR, DRX e Análise Térmica. As partículas que apresentaram as melhores formulações foram as de álcool polivinílico+alginato de sódio+ureia, pois apresentaram eficiência de incorporação próximas as das partículas de álcool polivinílico+alginato de sódio+caulinita intercalada, mas com maior estabilidade térmica, cerca de $200{ }^{\circ} \mathrm{C}$.

Palavras-chave: álcool polivinílico, alginato de sódio, ciclos de congelamento/descongelamento, incorporação de nitrogênio, ureia.

\begin{abstract}
In this work two nitrogen sources (urea and urea intercalated kaolinite) were incorporated in the polymer matrix polyvinyl alcohol and sodium alginate in the ratio of 3: 1 , using the methodology drip in $\mathrm{CaCl}_{2}$. The particles were subjected to freezing and subsequent thawing in order to improve the structure and thermal resistance of the polymer matrix. The particles were characterized by elemental analysis, FTIR, XRD, and thermal analysis. The particles that showed the best formulations were those of polyvinyl alcohol+sodium alginate+urea, because they showed the efficiency of incorporation nearby particles polyvinyl alcohol+sodium alginate+urea intercalated kaolinite, but with greater thermal stability, about $200^{\circ} \mathrm{C}$.
\end{abstract}

Keywords: polyvinyl alcohol, sodium alginate, cycles of freeze/thawing, incorporation of nitrogen, urea.

\section{Introdução}

Autilização de polímeros para encapsulamento de diversos materiais tem sido assunto de grande interesse, nas últimas décadas ${ }^{[1,2]}$. Dois polímeros amplamente utilizados para a incorporação de materiais são o álcool polivinílico (PVA) e o alginato de sódio (AS), por apresentarem baixa toxicidade e alta biodegradabilidade ${ }^{[1]}$. O PVAé um polímero sintético utilizado em uma ampla gama de aplicações industriais, comerciais, médicas e de alimentos ${ }^{[3]}$. É um polímero de baixo custo e boa estabilidade térmica, tendo o início de sua decomposição entre $230-290{ }^{\circ} \mathrm{C}^{[2,4,5]}$. O AS é um polímero linear polianiônico encontrado em algas castanhas ${ }^{[1]}$. Sua estrutura consiste em cadeias lineares de monômeros de ácido $\beta$-D-manurônico e de ácido $\alpha$-L-gulurônico, unidos por ligações tipo $(1 \rightarrow 4)$ em várias proporções ${ }^{[2,6]}$. Sua estabilidade térmica é de $210-240{ }^{\circ} \mathrm{C}^{[4,5,7,8]}$. O AS, pode sofrer o processo de gelificação ionotrópica na presença de íons bivalentes, como $\mathrm{Ca}^{2+}$, formando hidrogéis e, por isso, tem sido utilizado para produzir sistemas de liberação 
controlada de partículas de vários fármacos, proteínas e até mesmo células ${ }^{[1,6,9]}$.

Muitos estudos vêm sendo feitos na combinação de polímeros, que constitui um método muito útil para a melhoria ou a modificação das propriedades físico-químicas dos materiais poliméricos. Este processo gera misturas estruturalmente diferentes, afetando as propriedades mecânicas, a morfologia, a permeabilidade e degradação dos polímeros iniciais ${ }^{[10]}$. Outro procedimento de melhora de propriedades mecânicas, morfológicas e térmicas é o tratamento criogênico ${ }^{[11]}$. Os criogéis são formados como resultado do congelamento de polímeros, armazenamento no estado congelado por um tempo definido, e seguido de descongelamento ${ }^{[12]}$. Após os polímeros passarem por ciclos de congelamento/descongelamento (CC/D), há a formação de uma rede interpenetrante devido às ligações cruzadas, formando um emaranhamento de interações de hidrogênio entre as cadeias, o que é proporcionado pela aproximação das mesmas durante o processo de congelamento ${ }^{[13]}$. Este processo pode resultar na eliminação de água da matriz polimérica e redução do tamanho dos poros do material final[ ${ }^{[1]}$.

Um material novo a ser incorporado em matriz polimérica é a ureia. Molécula orgânica, nitrogenada, de ampla utilização na agricultura como fertilizante, dentre outras aplicações ${ }^{[14]}$. A ureia possui ponto de fusão de $133{ }^{\circ} \mathrm{C}$ e a sua decomposição térmica, muito complexa, inicia-se em cerca de $140{ }^{\circ} \mathrm{C}^{[15]}$. A ureia pode adquirir uma maior estabilidade térmica (cerca de 150-160 ${ }^{\circ} \mathrm{C}$ ) quando intercalada em caulinita $\left(\left[\mathrm{Si}_{4}\right] \mathrm{Al}_{4} \mathrm{O}_{10}(\mathrm{OH})_{8}\right)^{[16]}$, um argilomineral muito abundante no Brasil. Por ser um composto formado por camadas, a caulinita tem a capacidade de acomodar moléculas de ureia entre suas lamelas, atraves de ligações de hidrogênio, o que garante uma maior estabilidade térmica à ureia ${ }^{[16]}$. Em contrapartida, está comprovado que produtos de intercalação de ureia em caulinita são liberados rapidamente na presença de água ${ }^{[16]}$.

Pensando nisso, propõe-se a incorporação de ureia e caulinita intercalada com ureia, em partículas de PVA+AS através da técnica de gelificação ionotrópica seguido de criogenia, com o intuito de estudar a incorporação de ureia e a influência de CC/D neste processo.

\section{Experimental}

Inicialmente foi realizada a intercalação de ureia (marca Proquímios) em caulinita, proveniente do Rio Capim (Pará - Brasil) através do processo mecanoquímico, através do qual são misturadas ureia $(U)$ e caulinita $(C)$ na proporção $1: 4$, e moídas a seco com almofariz e pistilo de ágata por 7 horas $^{[17]}$.

\subsection{Elaboração das partículas de PVA +AS}

Preparou-se uma solução de PVA (marca Vetec, hidrólise de $86,5-89,5 \%)$ em água quente $\left(80{ }^{\circ} \mathrm{C}\right)$ e em seguida adicionou-se AS (marca Proquímios, viscosidade 520-580) na proporção de $3: 1(6 \% \text { de PVA e } 2 \% \text { de AS })^{[1]}$, a mistura ficou em agitação magnética por 12 horas. A solução de $\mathrm{PVA}+\mathrm{AS}$ foi aquecida a aproximadamente $80^{\circ} \mathrm{C}$ e gotejada através de uma bomba peristáltica da Tecnal, modelo
TE-BP-01-MINI, a 9,5 mL/min, em solução de $\mathrm{CaCl}_{2}$ a $5 \%{ }^{[1]}$ estando esta, em banho de gelo e sob agitação magnética ${ }^{[8]}$. As partículas formadas foram mantidas na solução de $\mathrm{CaCl}_{2}$ por 3 horas ${ }^{[18]}$.

Em seguida, as partículas foram separadas da solução de $\mathrm{CaCl}_{2}$ e parte delas, colocadas em estufa a $60{ }^{\circ} \mathrm{C}$ por 48 horas, resultando nas partículas de PVA+AS S CC/D. Outra parte das partículas foi submetida ao congelamento a $-18{ }^{\circ} \mathrm{C}$ por 48 horas e descongeladas a $25^{\circ} \mathrm{C}$ por 4 horas $^{[1]}$, o que corresponde a um CC/D. Após passarem por $\mathrm{CC} / \mathrm{D}$, as partículas também foram secas em estufa a $60{ }^{\circ} \mathrm{C}$ por 48 horas. Foram geradas partículas com $2 \mathrm{CC} / \mathrm{D}$ e com $4 \mathrm{CC} / \mathrm{D}$.

\subsection{Elaboração das partículas de PVA+AS+Cle $P V A+A S+U$}

As partículas de PVA+AS com caulinita intercalada $(\mathrm{PVA}+\mathrm{AS}+\mathrm{CI})$ e de $\mathrm{PVA}+\mathrm{AS}$ com ureia pura $(\mathrm{PVA}+\mathrm{AS}+\mathrm{U})$ foram elaboradas através da mesma metodologia descrita acima. Para as partículas de PVA+AS+CI foi adicionado CI à solução polimérica na proporção de 3:1:0,48, o que corresponde a $2,14 \%$ de ureia em relação a massa seca de reagentes. Já para as partículas de $\mathrm{PVA}+\mathrm{AS}+\mathrm{U}$ foi adicionado ureia pura, na proporção de 3:1:0,18, o que corresponde a 4,31\% de ureia em relação a massa seca de reagentes. Estas quantidades de CI e U foram definidas com base em testes realizados para verificar a consistência da solução formada, de maneira que concentrações mais elevadas de CI geram soluções muito espessas o que dificulta o gotejamento, pois obstruem as mangueiras. As soluções foram gotejadas a $8,0 \mathrm{~mL} / \mathrm{min}$ para $\mathrm{PVA}+\mathrm{AS}+\mathrm{CI}$, e a $9,5 \mathrm{~mL} / \mathrm{min}$ para $\mathrm{PVA}+\mathrm{AS}+\mathrm{U}$.

\subsection{Análise elementar}

Foi realizada num analisador elementar Elemental Analyser 2400 CHN Perkin-Elmer Series II - USA, utilizando em torno de $1,0 \mathrm{mg}$ da amostra pesado em balança analítica de 6 casas decimais.

\subsection{Especroscopia no Infravermelho com Transformada de Fourier (FTIR)}

As análises de FTIR foram realizadas em espectrômetro Perkin Elmer, modelo Frontier, utilizando partilhas de $\mathrm{KBr}$ obtidas por prensagem juntamente com a amostra. Para cada espectro são somadas 8 varreduras com resolução de $2 \mathrm{~cm}^{-1}$, na região de 4000 a $400 \mathrm{~cm}^{-1}$.

\subsection{Difratometria de Raios X (DRX)}

As amostras foram analisadas num difratômetro Bruker utilizando radiação $\mathrm{Cu} \mathrm{K \alpha}(\lambda=1,5418 \AA$ ) , com ângulos de varredura de 3 a $70^{\circ}$ de $2 \theta$, com passo de $0,05^{\circ}$ de $2 \theta$.

\subsection{Análise Termogravimétrica (TG) e Termogravimetria Derivada (DTG)}

Para a Análise Térmica foi utilizado um analisador térmico TA Instruments, modelo SDT Q600. As amostras foram aquecidas a uma razão de $10^{\circ} \mathrm{C} \cdot \mathrm{min}^{-1}$ de 30 a $800{ }^{\circ} \mathrm{C}$, sob atmosfera estática de ar com fluxo de $50 \mathrm{~mL} . \mathrm{min}^{-1}$. 


\section{Resultados e Discussão}

Na Figura 1 são apresentadas imagens das partículas de $\mathrm{PVA}+\mathrm{AS}+\mathrm{U}$ e PVA+AS+CI, ambas com 2 CC/D. Observa-se elevada semelhança entre as duas formulações de partículas, ambas com formato de "gota" devido a alta viscosidade das soluções que passaram pelo processo de gotejamento em solução de $\mathrm{CaCl}_{2}$.

\subsection{Análise elementar}

As porcentagens finais de ureia nas partículas de $\mathrm{PVA}+\mathrm{AS}+\mathrm{CI}$ e PVA+AS+U apresentadas na Tabela 1. As partículas de PVA+AS também foram analisadas, mas foi confirmada a ausência de nitrogênio, como esperado.

Observa-se que nem toda a ureia adicionada foi incorporada nas partículas. Essa perda nas partículas $\mathrm{S} C \mathrm{C} / \mathrm{D}$, tanto nas de $\mathrm{PVA}+\mathrm{AS}+\mathrm{U}$, quanto nas de $\mathrm{PVA}+\mathrm{AS}+\mathrm{CI}$, pode ter ocorrido no processo de produção das partículas, pois as mesmas são feitas através do gotejamento da solução polimérica em solução aquosa de $\mathrm{CaCl}_{2}$. Como a ureia é altamente solúvel em água, no momento em que as partículas são formadas, parte da ureia pode ter sido lixiviada pela solução.

Essa redução da quantidade de ureia incorporada também é observada com o aumento dos $\mathrm{CC} / \mathrm{D}$, fato que é devido à eliminação de água no processo de descongelamento das partículas. Quando a partícula é descongelada a água e/ou soluções aquosas presentes na estrutura, são eliminadas devido a aproximação e emaranhamento das cadeias de $\mathrm{PVA}^{[1]}$. Neste processo, a ureia por ser muito solúvel em água, pode ter sido carregada para fora da matriz polimérica,

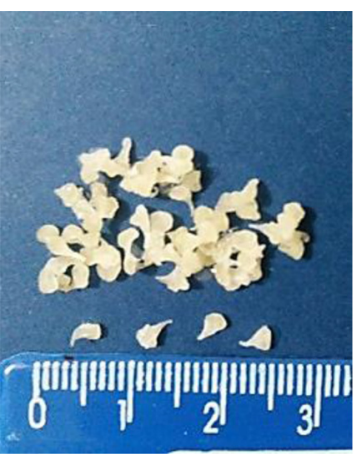

(A)

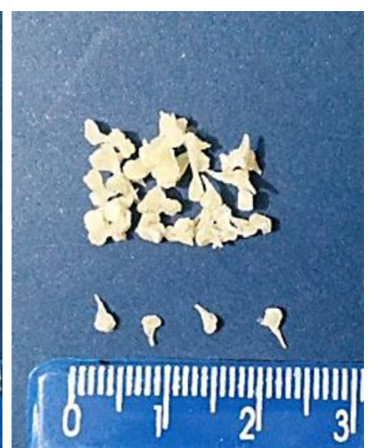

(B)
Figura 1. Fotografia de partículas de (A) $\mathrm{PVA}+\mathrm{AS}+\mathrm{U} \operatorname{com} 2 \mathrm{CC} / \mathrm{D}$ e (B) $\mathrm{PVA}+\mathrm{AS}+\mathrm{CI} \operatorname{com} 2 \mathrm{CC} / \mathrm{D}$.

Tabela 1. Porcentagens finais de ureia nas partículas de $\mathrm{PVA}+\mathrm{AS}+\mathrm{CI}$ e PVA+AS+U S CC/D, com 2 CC/D e com 4 CC/D.

\begin{tabular}{ccc}
\hline \multicolumn{2}{c}{ Tipo de partícula } & $\begin{array}{c}\text { Porcentagem } \\
\text { final de ureia na } \\
\text { partícula (\%) }\end{array}$ \\
\hline PVA+AS+U & S CC/D & 0,986 \\
2 CC/D & 0,761 \\
PVA+AS+CI & 4 CC/D & 0,407 \\
& S CC/D & 0,504 \\
& 2 CC/D & 0,311 \\
& 4 CC/D & 0,204 \\
\hline
\end{tabular}

sendo eliminada da partícula, gerando assim, uma diminuição da porcentagem de ureia incorporada.

\subsection{Especroscopia no Infravermelho com Transformada de Fourier (FTIR)}

A Figura 2A apresenta os espectros de infravermelho de amostras de caulinita, ureia, caulinita intercalada com ureia, AS e PVA. Observam-se para a caulinita pura, bandas relativas à deformação axial das hidroxilas externas em $3698 \mathrm{~cm}^{-1}$, e das hidroxilas internas em $3620 \mathrm{~cm}^{-1[17]}$. Para a ureia, observam-se duas bandas, em 3445 e $3348 \mathrm{~cm}^{-1}$, provenientes da deformação axial simétrica e assimétrica de N-H, respectivamente ${ }^{[19]}$. Após a intercalação de ureia na caulinita, a principal alteração ocorre no deslocamento das duas bandas características da ureia, para 3505 e $3388 \mathrm{~cm}^{-1}$. Essas novas bandas são associadas à ligação da molécula de ureia às hidroxilas externas da lamela da caulinita, o que é uma evidência do processo de interação ${ }^{[17]}$.

Tanto no espectro de PVA, quanto no de AS (Figura 2A), são observadas as bandas na região de $3400 \mathrm{~cm}^{-1}$, proveniente da deformação axial de $\mathrm{OH}$ associado, e em $2900 \mathrm{~cm}^{-1}$, devido a deformação axial da ligação C-H $\mathrm{H}^{[1,2,19]}$. Para o espectro de AS, observa-se ainda, a deformação do íon - $\mathrm{COO}^{-}$que dá origem a duas bandas, uma em $1415 \mathrm{~cm}^{-1}$ e outra de maior intensidade em $1610 \mathrm{~cm}^{-1}$, provenientes das deformações axiais simétrica e assimétrica, respectivamente ${ }^{[1,2,8,19,20]}$.

Para as partículas de PVA+AS (Figura 2B), observa-se que o emaranhamento das cadeias que ocorre no processo de elaboração das partículas não altera significativamente as estruturas dos polímeros envolvidos, pois os espectros das amostras de partículas apresentam as bandas nas mesmas regiões. As bandas nas regiões de 1415 e $1610 \mathrm{~cm}^{-1}$, provenientes da deformação simétrica e assimétrica do grupo funcional carboxílico, embora deslocadas para $1432 \mathrm{e} 1630 \mathrm{~cm}^{-1}$, ainda permanecem. Este deslocamento para frequências maiores é devido a reticulação com íons $\mathrm{Ca}^{2+}$, os quais interagem com os grupos funcionais carboxílicos unindo as cadeias de alginato, este resultado também foi encontrado na literatura ${ }^{[1]}$.

Através dos espectros de partículas de PVA+AS+CI (Figura 2C), pode-se observar que as mesmas conservaram as mesmas bandas características dos polímeros utilizados. Apresentam também, uma das bandas vistas anteriormente no espectro de infravermelho da CI (Figura 2Ab), na região de $3696 \mathrm{~cm}^{-1}$, relativa à deformação axial das hidroxilas externas. A banda em $826-827 \mathrm{~cm}^{-1}$ observada nas partículas de $\mathrm{PVA}+\mathrm{AS}$ e $\mathrm{PVA}+\mathrm{AS}+\mathrm{U}$, não é observada nas partículas de PVA+AS+CI, pois fica sobreposta pela mesma região de picos intensos da caulinita. Muitos picos característicos da CI ficaram encobertos pelos picos característicos dos polímeros, o que indica que a caracterização por FTIR não é suficiente para a comprovação da incorporação de nitrogênio nas partículas.

Nas partículas de PVA+AS+U (Figura 2D), a banda característica da deformação axial de $\mathrm{O}-\mathrm{H}\left(3400 \mathrm{~cm}^{-1}\right)$ é deslocada para frequências mais baixas com o aumento dos CC/D. Este deslocamento é decorrente do aumento de interações de hidrogênio intermoleculares, as quais interferem na força de ligação covalente-polar $\mathrm{O}-\mathrm{H}$, enfraquecendo-a, o que consequentemente interfere no sinal de FTIR detectado 


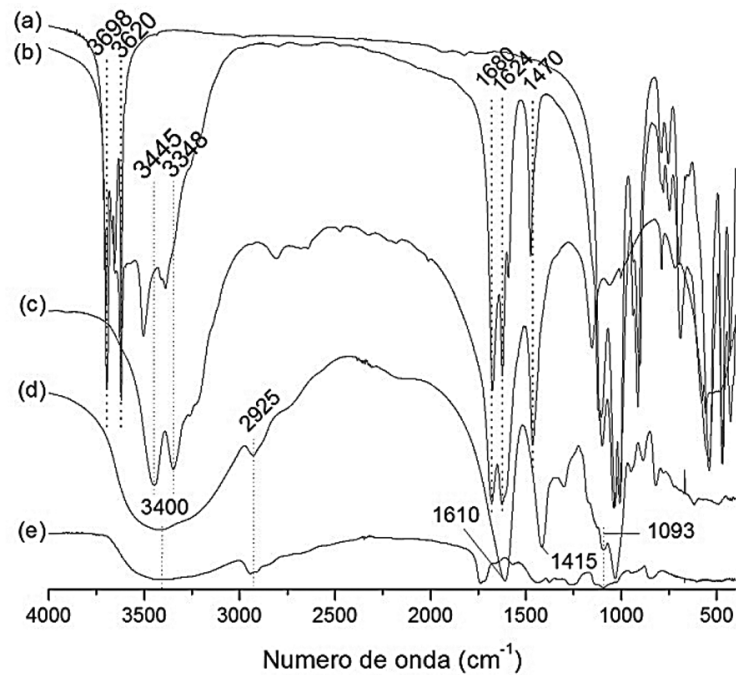

(A)

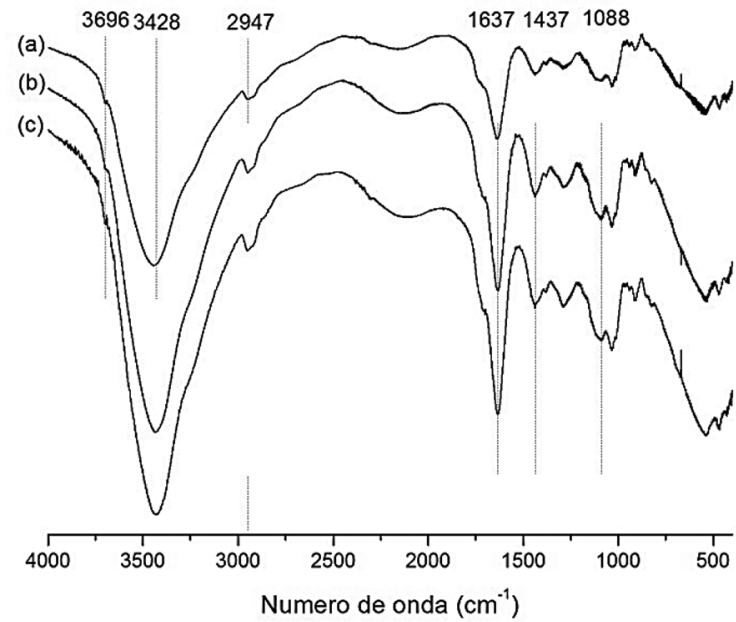

(C)

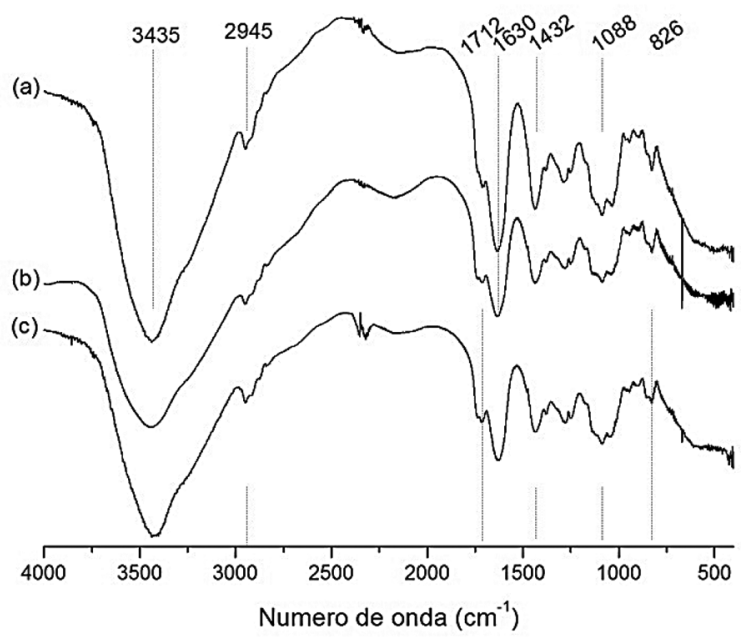

(B)

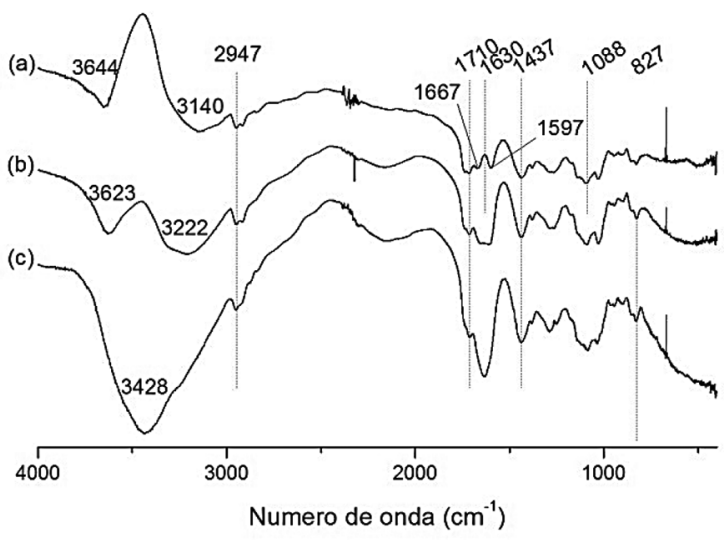

(D)

Figura 2. Espectros de infravermelho de amostras de (Aa) caulinita pura, (Ab) caulinita intercalada com ureia, (Ac) ureia pura, (Ad) alginato de sódio e (Ae) PVA; partículas de PVA+AS com (Ba) 4 CC/D, (Bb) 2 CC/D e (Bc) S CC/D; partículas de PVA+AS+CI com $(\mathrm{Ca}) 4 \mathrm{CC} / \mathrm{D}$, (Cb) $2 \mathrm{CC} / \mathrm{D}$ e (Cc) S CC/D; e partículas de PVA+AS+U, com (Da) $4 \mathrm{CC} / \mathrm{D}$, (Db) $2 \mathrm{CC} / \mathrm{D}$ e (Dc) S CC/D.

de forma a deslocar a banda para frequências menores ${ }^{[20]}$. Esse fato ocorre apenas nas partículas com ureia, a qual possui grandes possibilidades de se acomodar entre as cadeias poliméricas, devido ao seu tamanho reduzido, e formar ligações de hidrogênio com as hidroxilas dos polímeros, o que pode ter provocado o deslocamento da banda da região de $3400 \mathrm{~cm}^{-1}$. Este deslocamento se intensifica com o aumento dos $\mathrm{CC} / \mathrm{D}$, através dos quais há uma aproximação ainda maior das cadeias poliméricas, formando uma rede interpenetrante devido às interações de hidrogênio e consequente emaranhamento das cadeias de PVA $^{[1,13]}$. Este aumento de reticulação aumenta a aproximação das cadeias poliméricas com as moléculas de ureia, facilitando a formação de interações de hidrogênio.

Outra característica importante é a diminuição da intensidade da banda na região de $1630 \mathrm{~cm}^{-1}$ com o aumento dos CC/D e o surgimento de duas novas bandas em 1667 e $1597 \mathrm{~cm}^{-1}$, nas partículas com 4 CC/D. A banda na região de $1630 \mathrm{~cm}^{-1}$ é proveniente da deformação assimétrica de $-\mathrm{COO}^{-}$, o qual pode estar sofrendo influencia da presença de ureia na estrutura com o aumento da reticulação pelos CC/D. O surgimento das duas novas bandas pode estar relacionado com a formação de amidas secundárias, as quais apresentam sinal de dobramento $\mathrm{N}-\mathrm{H}$ na região de 1640-1550 $\mathrm{cm}^{-1[20]}$. Outra possibilidade é a formação de oximas ( $\mathrm{R}-\mathrm{CH}=\mathrm{N}-\mathrm{O}-\mathrm{H})$ as quais possuem absorção de $\mathrm{C}=\mathrm{N}$ na região de $1690-1640 \mathrm{~cm}^{-1}$, e ainda uma absorção larga de $\mathrm{O}-\mathrm{H}$ em $3650-2600 \mathrm{~cm}^{-1[20]}$. Este último fator, enquadra a absorção detectada em 3623 e $3644 \mathrm{~cm}^{-1}$ nas partículas de $\mathrm{PVA}+\mathrm{AS}+\mathrm{U}$ com 2 e $4 \mathrm{CC} / \mathrm{D}$, respectivamente ${ }^{[20]}$.

As partículas de $\mathrm{PVA}+\mathrm{AS}+\mathrm{U}$ foram as que apresentaram maior influencia dos $\mathrm{CC} / \mathrm{D}$ na sua reticulação e na sua estrutura, devido a contribuição da ureia na formação de interações de hidrogênio, possivelmente em vários pontos das cadeias poliméricas. 


\subsection{Difratometria de Raios X (DRX)}

Através da Figura 3A observa-se o pico de difração da caulinita em aproximadamente $12^{\circ}$ de $2 \theta$, que é característico da reflexão $001^{[16,21]}$. Já a ureia apresenta difração em aproximadamente $22^{\circ}$ de $2 \theta$. Após a intercalação, observa-se

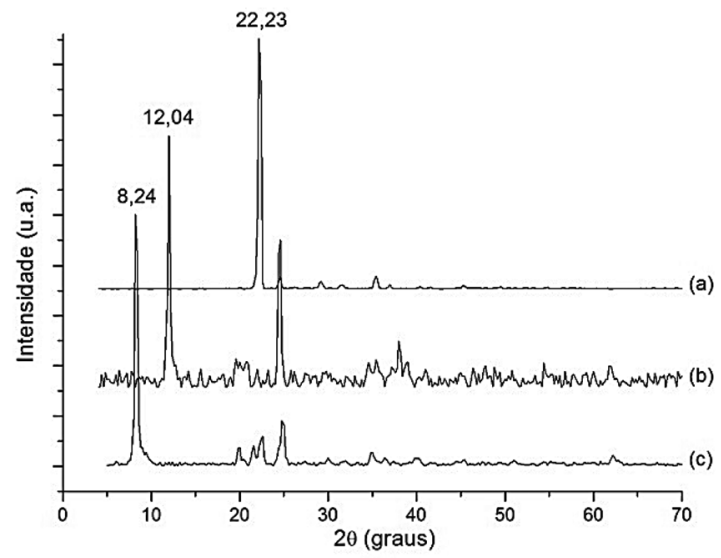

(A)

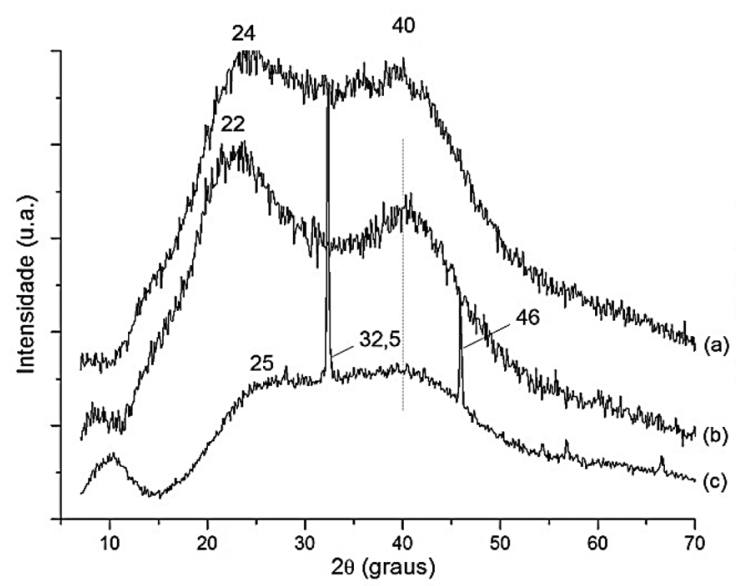

(C) o surgimento de uma difração em aproximadamente $8^{\circ}$ de $2 \theta$, característico da caulinita intercalada.

No difratograma de raios $\mathrm{X}$ de AS (Figura 3Ba) observam-se três picos característicos em, aproximadamente, $13,6^{\circ}$ de $2 \theta^{[1,4]}, 21,2^{\circ}$ de $2 \theta^{[4,7]}$ e $37,8^{\circ}$ de $2 \theta^{[7]}$ observados

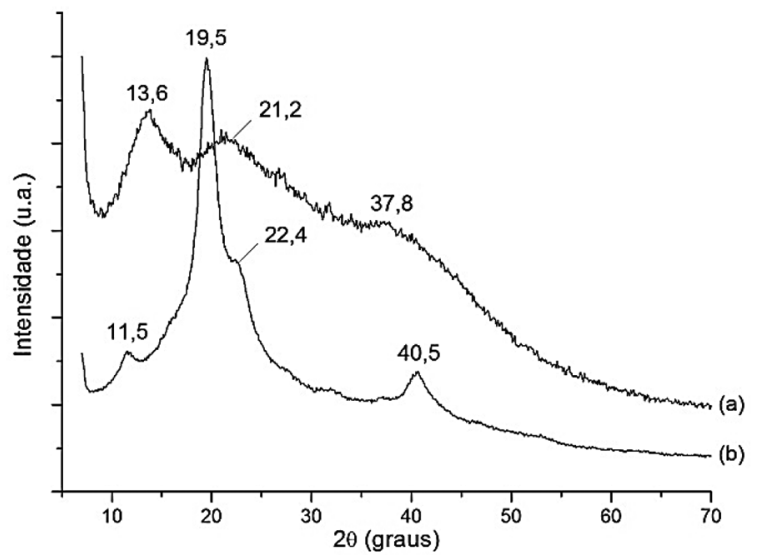

(B)

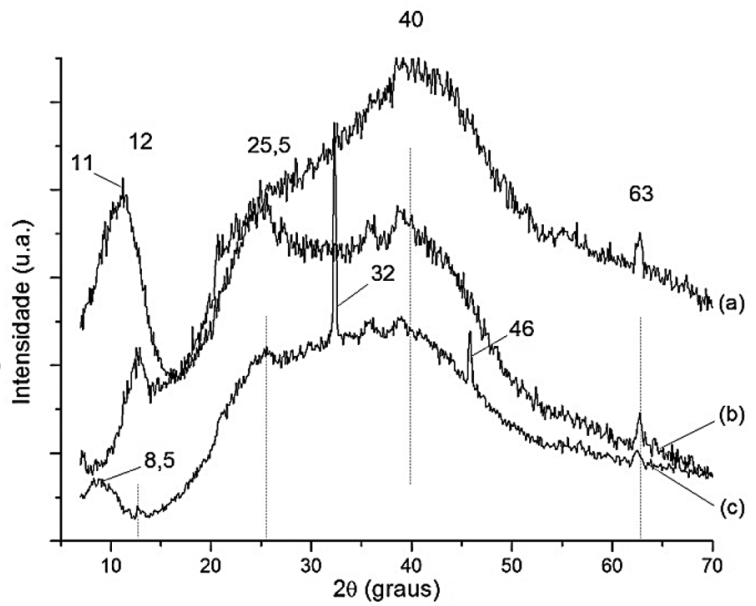

(D)

32

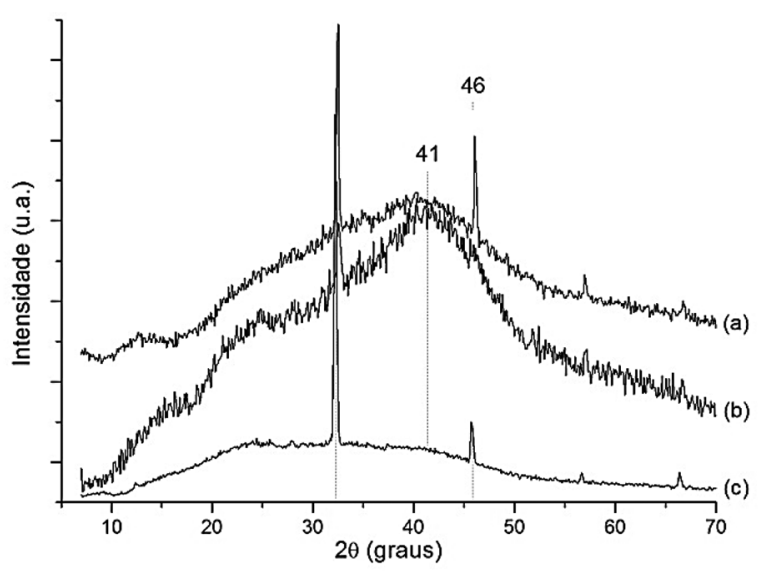

(E)

Figura 3. Difratogramas de Raios $X$ de amostras de $(\mathrm{Aa})$ ureia, $(\mathrm{Ab})$ caulinta e $(\mathrm{Ac})$ caulinita intercalada com ureia; $(\mathrm{Ba})$ alginato de sódio e (Bb) PVA; partículas de PVA+AS com (Ca) 4 CC/D, (Cb) 2 CC/D e (Cc) S CC/D; partículas de PVA+AS+CI com (Da) 4 CC/D, (Db) 2 CC/D e (Dc) S CC/D; e partículas de PVA+AS+U com (Ea) 4 CC/D, (Eb) 2 CC/D e (Ec) S CC/D. 
também, em outros trabalhos. Para o PVA (Figura 3Bb) observa-se picos de difração em $11,5^{\circ}$ de $2 \theta, 19,5^{\circ}$ de $2 \theta, 22,4^{\circ}$ de $2 \theta$ e $40,5^{\circ}$ de $2 \theta$, valores semelhantes aos encontrados em outros estudos ${ }^{[4,7,22]}$.

Os difratogramas de amostras de $\mathrm{PVA}+\mathrm{AS}$ com 4 e com 2 CC/D (Figura 3Ca,b) apresentam características muito semelhantes. Os picos de difração característicos do PVA apresentam-se mais largos devido à redução da cristalinidade. Isso se deve a possível formação de interações de hidrogênio entre os grupos hidroxila do PVA e grupos hidroxila ou carboxila do $\mathrm{AS}^{[2,4]}$. Ocorre a redução da cristalinidade com o aumento dos $\mathrm{CC} / \mathrm{D}$, devido ao aumento das ligações cruzadas $^{[23]}$. O não aparecimento dos picos na região de $32^{\circ}$ de $2 \theta$ e $46^{\circ}$ de $2 \theta$ nas amostras com CC/D pode ser devido à mudança de disposição das cadeias, ocorrida durante este processo.

Com relação às partículas de $\mathrm{PVA}+\mathrm{AS}+\mathrm{CI}$ (Figura 3D), pode-se observar que para às partículas sem $\mathrm{CC} / \mathrm{D}$ existe um pico na região de $8,5^{\circ}$ de $2 \theta$, região característica da presença de caulinita intercalada. Este pico existe apenas para as partículas sem $C C / D$, e para as partículas com $\mathrm{CC} / \mathrm{D}$ surge novamente um pico na região de $11^{\circ}$ de $2 \theta$ a $12^{\circ}$ de $2 \theta$, região característica da presença de caulinita pura. Isso indica que após os $\mathrm{CC} / \mathrm{D}$, parte da ureia foi removida dos espaçamentos interlamelares da caulinita restando quantidades indetectáveis por DRX. Este resultado é comprovado pela Análise Elementar, que comprova uma diminuição da quantidade de nitrogênio com o aumento dos CC/D.

Para as partículas de $\mathrm{PVA}+\mathrm{AS}+\mathrm{U}$ (Figura 3E) não é detectada a presença de ureia na estrutura, a qual apresentaria um pico na região de $22^{\circ}$ de $2 \theta$, apesar da Análise Elementar comprovar a existência de ureia nestas partículas. Assim como em FTIR, a técnica de difratometria de Raios X mostrou características diferentes para as partículas de $\mathrm{PVA}+\mathrm{AS}+\mathrm{U}$. Para as partículas com CC/D surgem os picos nas regiões de $32^{\circ}$ de $2 \theta$ e $46^{\circ}$ de $2 \theta$, os quais podem estar relacionados ao fato a ureia estar ligada as cadeias poliméricas através de interações de hidrogênio, o que já foi sugerido através dos resultados de FTIR, pois houve um deslocamento da banda característica da deformação axial de O-H (Figura 2D). Ainda pode estar relacionado com o surgimento dos dois novos picos no espectro de FTIR em $1667 \mathrm{e} 1597 \mathrm{~cm}^{-1}$, para as partículas de PVA+AS+U com 4 CC/D (Figura 2Da), os quais podem ter sido gerados pela formação de amidas secundárias, ou oximas, como discutido anteriormente.

\subsection{Análise Termogravimétrica (TG) e Termogravimetria Derivada (DTG)}

Todas as amostras de partículas, inclusive de amostras de $\mathrm{C}, \mathrm{U}, \mathrm{CI}$ e dos polímeros puros, foram submetidas à análise térmica e os resultados são apresentados na Tabela 2. Através dos resultados pode-se observar a temperatura inicial de decomposição de cada amostra, bem como a temperatura de decomposição máxima de cada etapa de decomposição. Para a CI, observa-se claramente que as três primeiras etapas de decomposição são referentes a ureia e a última etapa é referente a caulinita, indicando que houve a eliminação da matéria orgânica (ureia) sem a destruição da matriz em questão (caulinita) ${ }^{[17]}$. Resultados compatíveis a este trabalho foram encontrados na literatura para caulinita ${ }^{[17,21,24]}$, ureia $^{[15,17]}$, caulinita intercalada ${ }^{[17,24]}, \operatorname{PVA}^{[2,4,5]}$ e $\mathrm{AS}^{[5,7,8]}$.

A decomposição térmica das partículas ocorre em três etapas definidas pela DTG. As partículas de PVA+AS com $\mathrm{CC} / \mathrm{D}$ apresentam uma temperatura inicial de decomposição maior do que as partículas $\mathrm{S} \mathrm{CC} / \mathrm{D}\left(188^{\circ} \mathrm{C}>195^{\circ} \mathrm{C}>196^{\circ} \mathrm{C}\right)$. Este fato indica que os ciclos de $\mathrm{CC} / \mathrm{D}$ contribuem para uma leve melhora da estabilidade térmica do produto. Já as temperaturas de pico da curva DTG não apresentam aumento regular com o aumento dos CC/D.

Para as partículas de PVA+AS+CI observa-se um aumento na estabilidade térmica das partículas com o aumento dos $\mathrm{CC} / \mathrm{D}$, pois a temperatura de pico da curva de DTG da primeira etapa de decomposição aumenta com o aumento dos $\mathrm{CC} / \mathrm{D}\left(226,3{ }^{\circ} \mathrm{C}>235,8{ }^{\circ} \mathrm{C}>240,0{ }^{\circ} \mathrm{C}\right)$. Outro indício de aumento de estabilidade térmica é que quanto maior o número de $\mathrm{CC} / \mathrm{D}$, maior é a temperatura final de decomposição. Este aumento da resistência térmica com o aumento do número de $\mathrm{CC} / \mathrm{D}$, nos diferente tipos de partículas, pode ser resultado da formação de um emaranhado de cadeias de PVA, unidas por interações de hidrogênio, que foram facilitadas pela aproximação das mesmas durante o congelamento $^{[1]}$. Este fator dificulta a mobilidade das cadeias dos polímeros e a quebra de ligações.

$\mathrm{O}$ aumento de estabilidade térmica com o aumento de $\mathrm{CC} / \mathrm{D}$ também é observado para as partículas de PVA+AS+U. Isto é visível observando as temperaturas iniciais da primeira e terceira etapa de decomposição das partículas, as quais vão aumentando com o aumento dos CC/D. Este aumento também é observado nas temperaturas de decomposição da primeira etapa de decomposição. Outro indício é a temperatura final de decomposição, a qual aumenta com o aumento dos $\mathrm{CC} / \mathrm{D}\left(714^{\circ} \mathrm{C}>735^{\circ} \mathrm{C}>742{ }^{\circ} \mathrm{C}\right)$. Em comparação com as partículas de $\mathrm{PVA}+\mathrm{AS}+\mathrm{CI}$, as partículas de $\mathrm{PVA}+\mathrm{AS}+\mathrm{U}$ apresentam temperaturas de decomposição máxima maiores na primeira etapa de decomposição térmica. As temperaturas finais de decomposição são consideravelmente maiores para as partículas de $\mathrm{PVA}+\mathrm{AS}+\mathrm{U}$, indicando maior lentidão na decomposição térmica para estas partículas.

Este aumento de estabilidade com a adição de ureia pode estar relacionado ao fato de as moléculas de ureia estabelecerem interações de hidrogênio facilmente com as hidroxilas de grupamentos $-\mathrm{COOH}$ dos polímeros formadores das partículas. Já a caulinita, por apresentar estrutura volumosa, é acomodada na matriz polimérica com maior dificuldade, mesmo também apresentando grupos passíveis de interações de hidrogênio. $\mathrm{O}$ fato de a caulinita estar intercalada contribui para o aumento do espaço ocupado pela molécula na matriz polimérica, pois a intercalação aumenta o espaçamento basal entre as lamelas deste argilomineral. Portanto, pode-se dizer que dentre todas as partículas analisadas, as que apresentaram melhor estabilidade térmica foram as de $\mathrm{PVA}+\mathrm{AS}+\mathrm{U}$, fato que é intensificado pela presença dos CC/D. Observa-se na Tabela 2 que praticamente todas as amostras apresentam massa residual em temperatura de $800^{\circ} \mathrm{C}$, e com o aumento dos ciclos de congelamento/descongelamento a massa residual diminui. Em contrapartida, a estabilidade térmica em cada etapa de degradação aumenta quando o número de $\mathrm{CC} / \mathrm{D}$ é maior. 
Tabela 2. Valores dos intervalos de temperatura das etapas de decomposição (TG), temperatura de decomposição máxima de cada etapa de decomposição (DTG), perda de massa não cumulativa e total de amostras de C, U, CI, PVA, AS e das partículas de PVA+AS, $\mathrm{PVA}+\mathrm{AS}+\mathrm{CI}$ e $\mathrm{PVA}+\mathrm{AS}+\mathrm{U}, \mathrm{S} \mathrm{CC} / \mathrm{D}, \mathrm{com} 2 \mathrm{CC} / \mathrm{D}$ e com $4 \mathrm{CC} / \mathrm{D}$.

\begin{tabular}{|c|c|c|c|c|}
\hline Amostra & $\begin{array}{l}\text { Intervalo aproximado de } \\
\text { temperatura }(\mathrm{TG})\left({ }^{\circ} \mathrm{C}\right)\end{array}$ & $\begin{array}{c}\text { Temperatura de } \\
\text { decomposição máxima } \\
\text { (pico em DTG) }\left({ }^{\circ} \mathrm{C}\right)\end{array}$ & $\begin{array}{l}\text { Perda de massa não } \\
\text { cumulativa (\%) }\end{array}$ & Perda de massa total $(\%)$ \\
\hline $\mathrm{C}$ & $380,0-800,0$ & 494,4 & 14,87 & 83,28 \\
\hline \multirow[t]{4}{*}{$\mathrm{U}$} & $130,0-205,0$ & 175,5 & 75,87 & 0,527 \\
\hline & $205,0-250,0$ & 244,7 & 10,25 & \\
\hline & $250,0-300,0$ & 295,4 & 10,87 & \\
\hline & $300,0-370,0$ & 365,0 & 1,643 & \\
\hline $\mathrm{CI}(\mathrm{U})$ & $130,0-180,0$ & 161,0 & 6,554 & 65,08 \\
\hline $\mathrm{CI}(\mathrm{U})$ & $180,0-235,0$ & 212,3 & 11,10 & \\
\hline CI (U) & $235,0-250,0$ & 245,0 & 1,070 & \\
\hline $\mathrm{CI}(\mathrm{C})$ & $350,0-800,0$ & 494,4 & 13,43 & \\
\hline \multirow[t]{3}{*}{ PVA } & $214,0-379,0$ & 326,8 & 45,13 & 0,0 \\
\hline & $379,0-455,0$ & 415,0 & 32,29 & \\
\hline & $455,0-533,0$ & 479,6 & 20,02 & \\
\hline \multirow[t]{2}{*}{ AS } & $200,0-285,0$ & 241,7 & 36,32 & 20,97 \\
\hline & $535,0-578,0$ & 576,5 & 13,96 & \\
\hline \multirow[t]{3}{*}{$\mathrm{PVA}+\mathrm{AS} \mathrm{S} \mathrm{CC/D}$} & $188,0-371,0$ & 223,6 & 28,85 & 29,63 \\
\hline & $371,0-486,0$ & 432,6 & 14,58 & \\
\hline & $486,0-800,0$ & 518,4 & 16,47 & \\
\hline \multirow[t]{3}{*}{$\mathrm{PVA}+\mathrm{AS} 2 \mathrm{CC} / \mathrm{D}$} & $195,0-379,0$ & 236,2 & 33,63 & 23,16 \\
\hline & $379,0-477,0$ & 434,9 & 16,89 & \\
\hline & $477,0-800,0$ & 498,5 & 21,96 & \\
\hline \multirow[t]{3}{*}{$\mathrm{PVA}+\mathrm{AS} 4 \mathrm{CC} / \mathrm{D}$} & $196,0-376,0$ & 226,1 & 29,07 & 17,97 \\
\hline & $376,0-478,0$ & 431,1 & 13,48 & \\
\hline & $478,0-800,0$ & 519,1 & 20,60 & \\
\hline \multirow[t]{3}{*}{$\mathrm{PVA}+\mathrm{AS}+\mathrm{CI} \mathrm{S} C \mathrm{C} / \mathrm{D}$} & $183,0-366,0$ & 226,3 & 26,06 & 33,61 \\
\hline & $379,0-477,0$ & 432,8 & 10,99 & \\
\hline & $477,0-623,0$ & 505,6 & 14,08 & \\
\hline \multirow[t]{3}{*}{$\mathrm{PVA}+\mathrm{AS}+\mathrm{CI} 2 \mathrm{CC} / \mathrm{D}$} & $183,0-362,0$ & 235,8 & 26,30 & 31,49 \\
\hline & $362,0-470,0$ & 428,7 & 11,72 & \\
\hline & $470,0-636,0$ & 494,8 & 15,18 & \\
\hline \multirow[t]{3}{*}{$\mathrm{PVA}+\mathrm{AS}+\mathrm{CI} 4 \mathrm{CC} / \mathrm{D}$} & $191,0-367,0$ & 240,0 & 32,02 & 23,86 \\
\hline & $367,0-483,0$ & 433,5 & 17,74 & \\
\hline & $483,0-642,0$ & 520,7 & 17,36 & \\
\hline \multirow[t]{3}{*}{$\mathrm{PVA}+\mathrm{AS}+\mathrm{U} \mathrm{S} C \mathrm{C} / \mathrm{D}$} & $195,0-366,0$ & 243,7 & 26,85 & 29,95 \\
\hline & $366,0-473,0$ & 428,7 & 10,54 & \\
\hline & $473,0-714,0$ & 506,1 & 16,46 & \\
\hline \multirow[t]{3}{*}{$\mathrm{PVA}+\mathrm{AS}+\mathrm{U} 2 \mathrm{CC} / \mathrm{D}$} & $200,0-370,0$ & 245,6 & 31,35 & 22,82 \\
\hline & $370,0-477,0$ & 430,3 & 13,91 & \\
\hline & $477,0-735,0$ & 510,2 & 18,08 & \\
\hline \multirow[t]{3}{*}{$\mathrm{PVA}+\mathrm{AS}+\mathrm{U} 4 \mathrm{CC} / \mathrm{D}$} & $200,0-367,0$ & 251,2 & 29,10 & 26,61 \\
\hline & $367,0-471,0$ & 429,8 & 13,01 & \\
\hline & $471,0-742,0$ & 506,1 & 17,78 & \\
\hline
\end{tabular}

\section{Conclusões}

A incorporação de ureia em matriz de PVA+AS foi possível, sendo comprovado por análise elementar e DRX que a eficiência de incorporação diminui com o aumento do número de $\mathrm{CC} / \mathrm{D}$. As partículas de $\mathrm{PVA}+\mathrm{AS}+\mathrm{U}$ foram as que obtiveram maior alterações na matriz polimérica, maior estabilidade térmica, e elevaram a decomposição da ureia imobilizada a $200^{\circ} \mathrm{C}$, tendo em vista que a ureia pura se decompõe em aproximadamente $130{ }^{\circ} \mathrm{C}$. Além disso, apresentam a vantagem de serem mais viáveis economicamente, pois não apresentam caulinita em sua formulação. 


\section{Agradecimentos}

A CAPES pela concessão da bolsa de mestrado, a UTFPR e ao professor Fauze Jaco Anaissi da UNICENTRO, Campus Guarapuava - PR, pelas análises de DRX.

\section{Referências}

1. Hua, S., Ma, H., Li, X., Yang, H., \& Wang, A. (2010). pHsensitive sodium alginate/poly(vinyl alcohol) hydrogel beads prepared by combined $\mathrm{Ca}^{2+}$ crosslinking and freeze-thawing cycles for controlled release of diclofenac sodium. International Journal of Biological Macromolecules, 46(5), 517-523. http:// dx.doi.org/10.1016/j.ijbiomac.2010.03.004. PMid:20223260.

2. Islam, M. S., \& Karim, M. R. (2010). Fabrication and characterization of poly(vinyl alcohol)/alginate blend nanofibers by electrospinning method. Colloids and Surfaces. A, Physicochemical and Engineering Aspects, 366(1-3), 135140. http://dx.doi.org/10.1016/j.colsurfa.2010.05.038.

3. DeMerlis, C. C., \& Schoneker, D. R. (2003). Review of the oral toxicity of polyvinyl alcohol (PVA). Food and Chemical Toxicology, 41(3), 319-326. http://dx.doi.org/10.1016/S02786915(02)00258-2.

4. Li, W., Li, X., Chen, Y., Li, X., Deng, H., Wang, T., Huang, R., \& Fan, G. (2013). Poly(vinyl alcohol)/sodium alginate/ layered silicate based nanofibrous mats for bacterial inhibition. Carbohydrate Polymers, 92(2), 2232-2238. http://dx.doi. org/10.1016/j.carbpol.2012.12.004. PMid:23399282.

5. Yang, J. M., Wang, N. C., \& Chiu, H. C. (2014). Preparation and characterization of poly(vinyl alcohol)/sodium alginate blended membrane for alkaline solid polymer electrolytes membrane. Journal of Membrane Science, 457(1), 139-148. http://dx.doi.org/10.1016/j.memsci.2014.01.034.

6. Paula, H. C. B., Oliveira, E. F., Abreu, F. O. M. S., Paula, R. C. M., Morais, S. M., \& Forte, M. M. C. (2010). Esferas (beads) de alginato como agente encapsulante de óleo de croton zehntneri pax et hoffm. Polímeros, 20(2), 112-120. http://dx.doi.org/10.1590/S0104-14282010005000019.

7. Kuila, S. B., \& Ray, S. K. (2014). Dehydration of dioxane by pervaporation using filled blend membranes of polyvinyl alcohol and sodium alginate. Carbohydrate Polymers, 101, 1154-1165. http://dx.doi.org/10.1016/j.carbpol.2013.09.086. PMid:24299887.

8. Santagapita, P., Mazzobre, M., \& Buera, M. (2012). Invertase stability in alginate beads. Food Research International, 47(2), 321-330. http://dx.doi.org/10.1016/j.foodres.2011.07.042.

9. Turbiani, F. R. B., Kieckbusch, T. G., \& Gimenes, M. L. (2011). Liberação de benzoato de cálcio de filmes de alginato de sódio reticulados com íons cálcio. Polímeros, 21(3), 175-181. http:// dx.doi.org/10.1590/S0104-14282011005000034.

10. Zhu, G., Wang, F., Xu, K., Gao, Q., \& Liu, Y. (2013). Study on properties of Poly(vinyl alcohol)/Polyacrylonitrile blend film. Polímeros, 23(2), 146-151. http://dx.doi.org/10.4322/ polimeros.2013.076.

11. El-Hadi, A. A. (2003). Factors affecting the production of prednisolone by immobilization of Bacillus pumilus E601 cells in poly(vinyl alcohol) cryogels produced by radiation polymerization. Process Biochemistry, 38(12), 1659-1664. http://dx.doi.org/10.1016/S0032-9592(02)00102-4.

12. Lozinsky, V. I., Galaev, I. Y., Plieva, F. M., Savina, I. N., Jungvid, H., \& Mattiasson, B. (2003). Polymeric cryogels as promising materials of biotechnological interest. Trends in Biotechnology, 21(10), 445-451. http://dx.doi.org/10.1016/j. tibtech.2003.08.002. PMid:14512231.

13. Peppas, N. A. (1997). Hydrogels and drug delivery. Current Opinion in Colloid \& Interface Science, 2(5), 531-537. http:// dx.doi.org/10.1016/S1359-0294(97)80103-3.

14. Wang, Z., Gu, Z., Li, Z., Hong, Y., \& Cheng, L. (2013). Effects of urea on freeze-thaw stability of starch-based wood adhesive. Carbohydrate Polymers, 95(1), 397-403. http:// dx.doi.org/10.1016/j.carbpol.2013.02.009. PMid:23618285.

15. Schaber, P. M., Colson, J., Higgins, S., Thielen, D., Anspach, B., \& Brauer, J. (2004). Thermal decomposition (pyrolysis) of urea in an open reaction vessel. Thermochimica Acta, 424(1-2), 131-142. http://dx.doi.org/10.1016/j.tca.2004.05.018.

16. Budziak Fukamachi, C. R., Wypych, F., \& Mangrich, A. S. (2007). Use of $\mathrm{Fe}^{3+}$ ion probe to study the stability of ureaintercalated kaolinite by electron paramagnetic resonance. Journal of Colloid and Interface Science, 313(2), 537-541. http://dx.doi.org/10.1016/j.jcis.2007.04.078. PMid:17561069.

17. Gardolinski, J. E., \& Wypych, F. (2001). Esfoliação e hidratação da caulinita após intercalação com uréia. Química Nova,24(6), 761-767. Retirado em 15 Julho 2014, de http://www.scielo. br/pdf/qn/v24n6/6782.pdf

18. Cunha, M. A. A., Converti, A., Santos, J. C., Ferreira, S. T. S., \& Silva, S. S. (2009). PVA-hydrogel entrapped candida guilliermondii for xylitol production from sugarcane hemicellulose hydrolysate. Applied Biochemistry and Biotechnology, 157(3), 527-537. http://dx.doi.org/10.1007/s12010-008-8301-5. PMid:18633733.

19. Silverstein, R. M., Webster, F. X., \& Kiemle, D. J. (2007). Identificação espectrométrica de compostos orgânicos. Rio de Janeiro: LTC.

20. Pavia, D. L., Lampman, G. M., Kriz, G. S., \& Vyvyan, J. R. (2010). Introdução à espectroscopia. São Paulo: Cengage Learning.

21. Makó, E., Kristóf, J., Horváth, E., \& Vágvölgyi, V. (2009). Kaolinite-urea complexes obtained by mechanochemical and aqueous suspension techniques - a comparative study. Journal of Colloid and Interface Science, 330(2), 367-373. http://dx.doi. org/10.1016/j.jcis.2008.10.054. PMid:19019383.

22. Jia, X., Li, Y., Zhang, B., Cheng, Q., \& Zhang, S. (2008). Preparation of poly(vinyl alcohol)/kaolinite nanocomposites via in situ polymerization. Materials Research Bulletin, 43(3), 611-617.http://dx.doi.org/10.1016/j.materresbull.2007.04.008.

23. Marinho, J. R. D. (2005). Macromoléculas e polímeros. Barueri: Manole.

24. Orzechowski, K., Slonka, T., \& Glowinski, J.-J. (2006). Dielectric properties of intercalated kaolinite. Journal of Physics and Chemistry of Solids, 67(5-6), 915-919. http:// dx.doi.org/10.1016/j.jpcs.2006.03.001.

Enviado: Out. 15, 2014 Revisado: Maio 17, 2015 Aceito: Jun. 02, 2015 